\title{
A Note on Notation
}

Although this volume does not employ a great deal of esoteric notation, some of its conventions and symbols may be unfamiliar to the general reader and so require a few words of explanation.

1. A dagger $(t)$ identifies those languages, or language families, that are now extinct.

2. An asterisk $\left({ }^{*}\right)$ is used in two ways. Usually it identifies reconstructed (but historically unattested) words, e.g. Indo-European *akva-s 'horse.' The second use of the asterisk is restricted to Chapter 8 , where it identifies those languages whose sound system will in due course be found in Volume 2, e.g. * Iatmul.

3. A right arrow $(\rightarrow)$ is used to indicate that one language (or family) name has been replaced by another. For example, Barea $\rightarrow$ Nera means that the language frequently referred to as Barea is called Nera in this volume.

4. An equals sign $(=)$ is used to point out alternate language (or family) names that for reasons discussed in section 8.1 are sometimes better known than the name adopted here, e.g. Nama ( $=$ Hottentot).

5. Brackets ([ . . .]) enclose phonetic transcriptions, e.g. [dori] 'to wish for, desire.' In Chapter 8 the number of languages in any group is indicated in brackets, following the name of the group; e.g. CELTIC [4] means there are four extant Celtic languages.

6. Single quotes ('. . ') enclose English glosses of foreign words, e.g. French chien 'dog.'

7. Double quotes (". . ") are used to indicate direct quotation from a source or to call attention to a word or phrase, e.g. there are no "primitive" languages. 
8. Italics are used for non-English words (e.g. French chien 'dog'), for individual letters in running text (treated as phonetic elements, whether in English or not), and for simple emphasis (e.g. typological traits like gender).

9. Names of language groups are given in capital letters (e.g. SLAVIC, ROMANCE) in the lists and in the classification itself; names of single languages are in capital-and-lower-case letters (e.g. Russian, English, Ohlone). In the case of single languages that are themselves independent branches of a family, either notation may be used, e.g. ALBANIAN or Albanian.

10. The less-than sign $(<)$ indicates that a word, sound, or meaning derives historically from another, e.g. French chien $<$ Latin cane $(m)$; French $\check{s}$ $<$ Latin $k$.

11. The greater-than sign $(>)$ indicates that a word, sound, or meaning has changed into another over the course of time, e.g. Latin cane $(m)>$ French chien; Latin $k>$ French $\check{s}$.

12. The similar-to sign $(\sim)$ indicates either (a) that certain forms alternate morphologically in a language, e.g. English sing $\sim$ sang $\sim$ sung, IndoEuropean * ped- * pod- 'foot,' or (b) that variant forms of a root are found in different languages of a language family, e.g. Nilo-Saharan tok $\sim$ tek $\sim d i k$ 'one.'

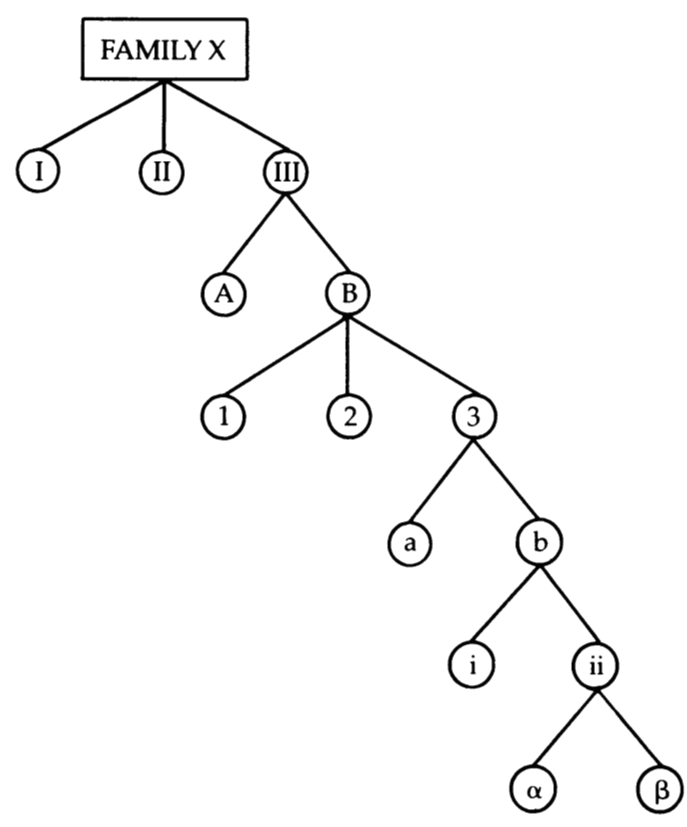




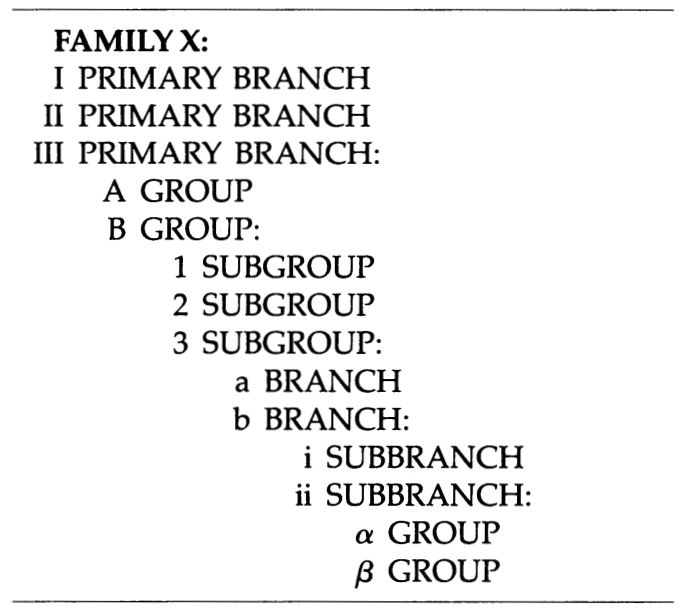

13. A hyphen (-) is sometimes used to separate a root from its affixes, either prefixes (e.g. English pre-fix) or suffixes (e.g. Greek dído-mi 'I give'). It is also used with prefixes and suffixes by themselves (e.g. the English prefix pre-, the Greek suffix - $m i{ }^{\prime} \mathrm{I}^{\prime}$ ) or following a root (e.g. Indo-European *ped- * pod- 'foot').

14. The system of representing genetic tree structure is explained in section 8.2, but because such trees occur in lists throughout the book, a brief outline of the notation is given here. Genetic classifications are often represented in terms of branching diagrams, as in the figure on the facing page.

In this book such structures are represented by a system of indentation, where each successive level of branching is indicated by indentation to the right and each node is preceded by a letter or number to identify the taxonomic level of the group. Accordingly, the branching-tree diagram in the figure will have the representation shown in the list above. 

A Guide to the World's Languages

\author{
VOLUME 1: CLASSIFICATION
}


